\title{
FOREWORD \\ SPECIAL ISSUE DEDICATED \\ TO SELECTED SURVEYS IN NONLINEAR PROGRAMMING
}

\author{
E.G. Birgin ${ }^{1}$, N. Krejić ${ }^{2}$ J.M. Martínez ${ }^{3}$, and M. Raydan ${ }^{4}$
}

Operations Research involves the sciences and techniques associated with decision making problems. Making decisions usually requires optimization (maximizing utilities and profits or minimizing loss), risk analysis needs careful identification of objectives and restrictions, and every mathematical model of real-life systems requires fitting parameters before its actual application.

In this issue several carefully selected survey papers tackle different areas of Continuous Optimization. By this we mean that decisions take place in the continuous world, in contrast to Discrete Optimization, that deals with discrete variables. The survey papers are written by worldwide experts in every one of the considered topics.

Nataša Krejić and Nataša Krklec Jerinkić review stochastic gradient methods for unconstrained optimization and Clovis Gonzaga and Elizabeth Karas discuss the complexity of first-order methods for smooth convex minimization. Classical smooth optimization algorithms are addressed by Margherita Porcelli and Stefania Bellavia under the preconditioning perspective, and by Sandra Santos who surveys trust-region methods.

Stabilized sequential quadratic programming methods for optimization with nonlinear constraints are surveyed by Damián Fernández and Mikhail Solodov. The nonlinearly constrained optimization problem is also addressed by Roberto Andreani and Paulo Silva in their study on constant rank constraint qualifications, and by Walter Gómez-Bofill and Juan Gómez in their review on linear and nonlinear semidefinite programming.

Andreas Fischer, Markus Herrich, and Klaus Schönefeld, on the one hand, and Joaquim Júdice, on the other hand, consider the related problems of Generalized Nash Equilibrium and Optimization with Linear Complementarity constraints.

\footnotetext{
${ }^{1}$ Department of Computer Science IME-USP, University of São Paulo, Rua do Matão 1010, Cidade Universitária, 05508090 São Paulo SP, Brazil. E-mail: egbirgin@ime.usp.br

${ }^{2}$ Department of Mathematics and Informatics, University of Novi Sad, Trg Dositeja Obradovića 4, 21000 Novi Sad, Serbia. E-mail: natasak@uns.ac.rs

${ }^{3}$ Department of Applied Mathematics, IMECC-UNICAMP, State University of Campinas, CP 6065, 13081-970 Campinas SP, Brazil. E-mail: martinez@ime.unicamp.br

${ }^{4}$ Departamento de Cómputo Científico y Estadística, Universidad Simón Bolívar, Ap. 89000, Caracas 1080-A, Venezuela. E-mail: mraydan@usb.ve
} 
Finally, Ellen Fukuda and Luis Mauricio Graña Drummond review descent methods for multiobjetive optimization, Andreas Griewank addresses automatic differentiation, emphasizing its relation with linearization and non-smooth optimization, and Welington de Oliveira and Claudia Sagastiábal address non-smooth optimization under the bundle point of view.

We would like to thank the authors that submitted their surveys to this special issue, the anonymous referees that reviewed the submitted articles in detail, and the editor of Pesquisa Operacional and the Brazilian Society of Operations Research that made it possible the edition of this issue. 